\title{
Green palmar discoloration in a patient with primary sclerosis cholangitis: eccrine chromhidrosis or pompholyx?
}

\author{
Bochra Bouchabou ${ }^{1}$, Fatma Ben Farhat ${ }^{1}$, Rym Ennaifer ${ }^{1}$, Abdelwaheb Ennakhli ${ }^{1}$, and \\ Houda Ben nejma ${ }^{1}$ \\ ${ }^{1}$ University Hospital Center Mongi Slim
}

April 7, 2021

\begin{abstract}
Chromhidrosis is defined by the secretion of colored sweat and pompholyx is a type of eczema characterized by the development of palmar and plantar vesicles. The case that we report can be eccrine chromhidrosis resembling the clinical features of pompholyx with explanation of probable pathomechanism.
\end{abstract}

Green palmar discoloration in a patient with primary sclerosis cholangitis: eccrine chromhidrosis or pompholyx?

Bochra Bouchabou1,2 | Fatma Ben Farhat1,2 | Rym Ennaifer 1,2| Abderrahmen Ennakhli1,2 | Houda Ben Nejma1,2

1Department of Gastroenterology, Mongi Slim Hospital, Tunis, Tunisia

2Faculty of Medicine of Tunis, University of Tunis El Manar, Tunis, Tunisia

Correspondence

Fatma Ben Farhat, Department of Gastroenterology, Mongi Slim Hospital, Tunis, Tunisia. Email Fatmabf8@gmail.com

Abstract:

Background: Chromhidrosis is defined by the secretion of colored sweat. As sweat can be produced by either eccrine or apocrine glands, chromohidrosis can be divided into subtypes and can have multiple locations through the body. In the other hand, pompholyx is a type of eczema characterized by the development of palmar and plantar vesicles.

Case report: we report the case of a 33-year-old woman with decompensated cirrhosis complicating Primary sclerosis cholangitis (PSC) suffering from abnormal bilateral pigmentation on palms, which lasted two weeks. On clinical examination, green desquamative papules were observed on the two palms (Fig.1) and levels of total $(34.4 \mathrm{mg} / \mathrm{dl})$ and direct $(17 \mathrm{mg} / \mathrm{dl})$ bilirubin were elevated. No specimen for histological research was done because lesions were already desquamative. Bacterial samples were done without any sign of fungic or bacterial infection.Conclusion: The case that we report can be eccrine chromhidrosis resembling the clinical features of pompholyx with explanation of probable pathomechanism. The green color is attributable to the switch from brown-colored bilirubin to green-colored biliverdin by oxidative processes.

KEYWORDS:

Primary sclerosis cholangitis, Green palmar Discoloration, eccrine chromhidrosis, pompholyx 


\section{Key Clinical Message:}

There are various causes of cutaneous green discoloration including granulocytic sarcoma, poisoning, acute pancreatitis, eccrine chromhidrosis and chronic liver disease. To our knowledge, we report the first case of acral green pigmentation:

Introduction:

Primary sclerosis cholangitis (PSC) is an autoimmune disease of the liver characterized by a progressive course of cholestasis with inflammation and fibrosis of the intrahepatic and extrahepatic bile ducts that can associated to hyperbilirubinemia in complicated or advanced stage. The spontaneous appearance of green discoloration of the hair, skin, and nails is unusual $(1,2)$. There are various causes of cutaneous green discoloration. Causes are multiple include granulocytic sarcoma, poisoning, acute pancreatitis, eccrine chromhidrosis and chronic liver disease.

\section{Case report}

A 33-year-old woman was examined for abnormal bilateral pigmentation on palms, which lasted two weeks. She was a housewife and she had decompensated cirrhosis complicating PSC as underlying diseases. She was hospitalized a month before for severe iron deficiency anemia with Hemoglobin level at $5.5 \mathrm{~g}$ per dl without externalized bleeding. She had a blood transfusion with a non-hemolytic febrile reaction. This episode was jugulated after stopping transfusion. She had intravenous iron replacement cure. On clinical examination, green desquamative papules were observed on the two palms (Fig.1) and levels of total (34.4 mg/dl) and direct $(17 \mathrm{mg} / \mathrm{dl})$ bilirubin were elevated. No specimen for histological research was done because lesions were already desquamative. Bacterial samples were done without any sign of fungic or bacterial infection. Hence, the patient was treated with emollient.Abnormal pigmentation was almost resolved, despite of a persistent high level of total $(40 \mathrm{mg} / \mathrm{dl})$ and direct $(22 \mathrm{mg} / \mathrm{dl})$ bilirubin. Patient died after three weeks from severe hepatic encephalopathy and general state alteration.

\section{Discussion}

The spontaneous appearance of green discoloration of the hair, skin, and nails is unusual $(1,2)$. There are various causes of cutaneous green discoloration. Differential diagnoses include granulocytic sarcoma, poisoning (such as by arsenic), acute pancreatitis (Grey Turner and Cullen signs with the appearance of ecchymotic patches on the abdomen), eccrine chromhidrosis (which may cause yellow, blue, black, or green discoloration) and chronic liver disease. Hair and nails can become green from the deposition of copper or from Pseudomonas infection. Other causes of green-pigmented lesions, including pseudomonas infection and exogenous staining that can be excluded by negative Gram stain result and the absence of greenish substance exposure, respectively (3). Green pigmentation on the palms and soles in patients with hyperbilirubinemia is a rare condition, with only 6 reported cases in the literature (1,3-6). Previous articles have reported that the green pigmentations are mainly along dermatoglyphic ridges where the eccrine sweat glands open $(4,6,7,9)$. The etiology of pompholyx is unknown, but associations with atopy and contact sensitivity have been reported $(2,7,8)$. We describe an unusual case of pompholyx presenting as green palmar vesicles in a patient presenting PSC in terminal cirrhosis stage with severe hyperbilirubinemia. Although pompholyx is spongiotic dermatitis, the greenish discoloration of the palmoplantar vesicles seen in our patient cannot be explained by spongiotic changes alone. We hypothesize that this condition occurs because increased water-soluble bilirubin is delivered to the intraepidermal sweat glands of patients with hyperbilirubinemia, where it is deposited into the stratum corneum $(1,2,4)$. Because of the high density of eccrine sweat glands in the palm and sole, pompholyx was initially regarded as being associated with disordered sweat ducts. The vesicular eruptions were thought to arise primarily in intra-epidermal sweat ducts, accompanied by hyperhidrosis (8). Pathogenetically, pompholyx was regarded as arising because of excessive secretion of sweat and dilatation of the intra-epidermal portion of the eccrine sweat ducts (8). The involvement of sweat units in the pathogenesis of pompholyx was disputed in reports describing the development of palmar and plantar vesicles independent of sweat ducts (8). In addition, considering the role of contact allergens in the pathogenesis of pompholyx, bile components, such as bilirubin, in sweat, may have acted as sensitizers, 
exacerbating the inflammatory spongiosis (8). In summary, although sweat units are not involved in the development of pompholyx, secondary pathological damage to the ducts may be driven by inflammatory spongiosis. Such involvement of sweat ducts in the pathogenesis of pompholyx may explain the bile-coloured vesicles of our patient. In any patient with hyperbilirubinemia and palmoplantar vesicles, the differential diagnosis should include eccrine chromhidrosis, a rare condition in which pigment from dyes or medications are excreted via the eccrine sweat glands $(2,3,9)$. Three subtypes have been described by Cilliers and de Beer: true eccrine chromhidrosis, pseudo-eccrine chromhidrosis, and apocrine chromhidrosis $(10,11)$. Pseudoeccrine chromhidrosis occurs when the colorless sweat gets colored on reaching the skin on reaction with exogenous chromogenic bacterial products such as Corynebacterium, piedraia, bacillus species, chemicals, paints, and dyes $(10,12)$. True eccrine chromhidrosis is a less common generalized disorder mostly caused by coloring of the clear eccrine sweat by dyes, pigments, or metals(10). There is a paucity of data regarding the eccrine chromhidrosis in medical literature. The cases of eccrine chromhidrosis to the best of our knowledge reported till date are summarized in Table 1.

In addition to these features, intraepidermal spongiosis and vesiculation can be seen when eccrine chromhidrosis presents as pompholyx, as seen in our case. Majority of patients had hyperbilirubinemia secondary to either liver disease, cholelithiasis, or cancerous processes. It is possible that in our case, because the green vesicles were located on the palms, where the eccrine sweat gland density is highest, and along the dermatoglyphic ridges, our patient's presentation may actually be a form of eccrine chromhidrosis. This possibility is supported by proposed pathophysiology that direct bilirubin is water soluble and may serve as the pigment or stain that is necessary in the pathologic basis of chromhidrosis $(2,10,12)$. Kanzaki and Tsuda (4) reported two cases of eccrine chromhidrosis with liver disease. In hepatocytes associated with liver disease, bile may become pigmented with brown color. No histological examination had been done because the patient was seen for a late stage of the eruption. Indeed, the patient already had desquamative lesions. The bile pigment may be difficult to highlight because can be washed out during histological fixation if it is located within the spaces of the eccrine ducts and vesicles, and not in the cellular spaces $(2,10)$. Possible pathomechanisms of three essential factors that could contribute towards the development of pigmentation: (1) increased plasma level of water-soluble direct bilirubin, (2) high fever with sweating, and (3) a thick horny layer. The green color is attributable to the switch from brown-colored bilirubin to green-colored biliverdin by oxidative processes.

\section{Conclusion:}

The case that we reported can be eccrine chromhidrosis resembling the clinical features of pompholyx with explanation of probable pathomechanism. To our knowledge, we report the first case of acral green pigmentation in a patient with PSC.

ACKNOWLEDGMENT: Published with written consent of the patient.

CONFLICTS OF INTEREST : None declared.

AUTHOR CONTRIBUTIONS : BFF and BB: wrote the manuscript. ER and EA: held the patient in the department. BNH: reviewed the manuscript.

\section{Bibliography:}

1. Allegue F, Hermo JA, Fachal C, Alfonsi'n N. Localized green pigmentation in a patient with hyperbilirubinemia. J Am Acad Dermatol. juill 1996;35(1):108-9.

2. Uzoma M, Singh G, Kohen L. Green palmoplantar vesicular eruption in a patient with hyperbilirubinemia. JAAD Case Rep. juill 2017;3(4):273-5.

3. Triwongwaranat D, Kasemsarn P, Boonchai W. Green Pigmentation on the Palms and Soles. JAMA Dermatol. 1 nov 2013;149(11):1339.

4. Kanzaki T, Tsuda J. Bile pigment deposition at sweat pores of patients with liver disease. J Am Acad Dermatol. avr 1992;26(4):655-6. 
5. Fung MA, LeBoit PE, Maibach HI. Coalescing green and yellow papules on the feet. Arch Dermatol. janv 2000;136(1):113, 116.

6. Di Stefani A, Bianchi L, Orlandi A, Chimenti S. Green sweating spots on hands and feet: unusual expression of hyperbilirubinemia. Ann Intern Med. 7 nov 2006;145(9):710-1.

7. Handa S, Jindal R, Kaur I, Gupta T. Hand eczema: Correlation of morphologic patterns, atopy, contact sensitization and disease severity. Indian J Dermatol Venereol Leprol. 2012;78(2):153.

8. Lee W, Lee D, Kim C, Won C, Chang S, Lee M, et al. Pompholyx with bile-coloured vesicles in a patient with jaundice: are sweat ducts involved in the development of pompholyx? J Eur Acad Dermatol Venereol. fevr 2010;24(2):235-6.

9. Keum DI, Hong H, Lee S-H, Ahn SK. Eccrine Chromhidrosis Resembling Clinical Features of Pompholyx with Bile-Like Greenish Pigmentation on the Right Palm and Soles. Ann Dermatol. 2015;27(4):482.

10. Jaiswal A, Ravikiran S, Roy P. Red eccrine chromhidrosis with review of literature. Indian J Dermatol. 2017;62(6):567.

11. Cilliers J, de Beer C. The Case of the Red Lingerie - Chromhidrosis Revisited. Dermatology. 1999;199(2):149-52.

12. Koley S, Mandal RK. Red and Black Pseudochromhidrosis. Indian J Dermatol. aout 2016;61(4):454-7.

13. Kawakami M, Hidano A. Skin eruption in a patient with hepatitis A. In (Tokyo); 1989. p. 593-5.

14. Krishnaram A, Bharathi S, Krishnan S. An interesting case of bisacodyl (dulcolax)-induced chromhidrosis. Indian J Dermatol Venereol Leprol. 2012;78(6):756.

15. Ghosh SK, Rudra O, Kar R, Ghosh A, Agarwal M. A curious case of blue-green discoloration in a middle-aged indian man: Chromhidrosis. Dermatol Online J. 18 nov 2015;21(11).

\section{List of figures and tables:}

Fig 1. Multiple deeply seated vesicles with greenish hue with accentuation of the dermatoglyphic lines on the palms

Table 1: Literature review of eccrine chromhidrosis

\section{Hosted file}

Fig 1.pdf available at https://authorea.com/users/406190/articles/516945-green-palmardiscoloration-in-a-patient-with-primary-sclerosis-cholangitis-eccrine-chromhidrosis-orpompholyx 